\title{
Service-Oriented Publish/Subscribe Architecture for Large Scale Wireless Sensor Networks
}

\author{
CHEN Jin-hui \\ School of Computer \& Software \\ Nanjing University of Information Science \& Technology \\ Nanjing, China \\ cjh@nuist.edu.cn
}

\begin{abstract}
Large scale wireless sensor networks (LSWSNs) need to provide complete solutions for users by integrating services and data into information service systems, and are more focused on their own functions. Services are playing more and more important role in LSWSNs. This paper presented an approach, called SOPSA, for modeling and implementing the architecture of LSWSNs applications using a service-oriented model. Considering bridging the gap between sensor nodes and enterprise applications where sensor data is used, SOPSA was defined by integrated LSWSNs middleware with publish/subscribe(Pub/Sub). SOPSA was composed of LSWSNs gateway, middleware, and application service. SOPSA management is designed through five function aspects, such as registration, library, routing, subscription and publishing. The analysis indicated that the middleware made SOPSA better suited to the distributing environment with multi-domain registry, and was able to support and integrate a variety of service discovery and composition. The results imply that SOPSA can be realized effectively.
\end{abstract}

Keywords-service; publish/subscribe; wireless sensor networks; architecture; model

\section{INTRODUCTION}

Services are playing more and more important role in LSWSNs: LSWSNs provide complete solutions for users by integrating services and data into information service systems, and are more focused on their own functions. $\mathrm{Pub} / \mathrm{Sub}$ is an asynchronous communication paradigm that supports many-to- many interactions between a set of clients. A client can be an information publisher, an information subscriber, or both. Client interactions are data-centric: publishers describe their publishable events, subscribers express their interest in events, and the Pub/Sub protocol delivers the published events to their corresponding event subscribers. SOPSA for LSWSNs is a kind of architecture that makes use of the Pub/Sub paradigm to provide the desired services for sensing-based pervasive computing applications. The motivation behind SOPSA derives from the gap between the high-level requirements from pervasive computing applications and the complexity of the operations in the underlying LSWSNs.

Alkhatib explored the wireless sensor networks(WSNs) architecture according to the open system interconnection model with some protocols in order to achieve good background on the WSNs[1]. Celandroni provided a survey on both sensor and satellite state-of-the-art technologies, with the aim of identifying the best-suited configurations matching requirements associated to each of the aforementioned applications[2]. Alonso implemented a hardware-embedded reactive agents platform based on a service-oriented architecture over heterogeneous WSNs[3]. Karakostas took a different approach-deploying interoperable SOAP-based web services directly on the nodes to bridge the gap between sensor nodes and enterprise applications such as factory monitoring, control, and tracking systems where sensor data is used[4]. Mottola introduced a taxonomy of WSNs programming approaches that captures the fundamental differences among existing solutions[5]. Lounis proposed an effective and flexible security mechanism that guarantees confidentiality, integrity as well as fine grained access control to outsourced medical data. This mechanism combines several cryptographic schemes to achieve high flexibility and performance[6]. Gholami evaluated the performance of three types of techniques, namely neural based, Kalman filter based and trilateration based techniques, having been proposed to tackle the problem of real-time mobile sensor node tracking in a wireless sensor network with passive architecture[7]. Ahmad proposed a model for the disaster survivor detection based on extremely critical disaster situation where this energy efficient architecture can successfully trace and locate thousands of people in critical circumstances[8]. Egbogah explored the current state of the art in medium access control protocols for WSNs, and sought an energy efficient solution that is robust to mobility and delivers data in a timely fashion[9]. Garay began with an objective description of the RF architecture and with the implementation of a RSSI analysis in real scenarios, and presented an architecture for WSNs operating in the $2.4 \mathrm{GHz} \mathrm{RF}$ band for implementation in environments of small tunnels[10]. Koo presented multi-sensor centric smart sensor network architecture using general mobile devices in order to provide more efficient and valuable sensor network application and services. The proposed system architecture is based on IEEE 802.15.4-2006 standard with smart mobile devices[11]. Lamont presented a tiered sensor networking architecture that employs 
advanced WSNs technologies for military operations, this architecture results in an agile surveillance system with a focus on improved operational flexibility and usability[12]. Hackmann proposed a cyber-physical co-design approach to structural health monitoring based on WSNs. The approach closely integrates an energy effcient, multi-level computing architecture specically designed to leverage the multi-resolution feature of the exibility-based approach [13]. In terms of the LSWSNs architecture, people have done a lot of work. But the functional requirements based on service contents often change, and user's needs are different. These make the service-oriented Pub/Sub systems efficiently publishing and subscribing remain still an open issue.

This paper presents the model and system design that tailor SOPSA to LSWSNs environment. Specifically, it makes the following primary contributions. Firstly, we propose SOPSA based on LSWSNs, and define its architecture. And secondly, we design and implement SOPSA management, such as registration, library, routing, subscription and publishing.

\section{SOPSA MODEL}

SOPSA establishes an intelligent processing system to cover LSWSNs, including information collection, information exchange and information services, and other functions. Its specific objectives are as follows. SOPSA establishes a data collection and aggregation system to support different manufacturers use different sensors, and gathers its sensing data to realize data center aggregation. SOPSA establishes a unified information collection and exchange platform for application systems to share sensing information. SOPSA provides data support for information integration and collaboration running between applications or within applications. SOPSA integrates P/S technology into LSWSNs applications, deploys LSWSNs service applications along with any combination of other services, and implements the separation of service providers from service subscribers. These components, the specific model and interface constitute SOPSA shown in Fig .1.

\begin{tabular}{|c|c|c|c|}
\hline application & \multicolumn{2}{|c|}{ application $\mathrm{j}$} & $\begin{array}{c}\text { application } \\
\text { service layer }\end{array}$ \\
\hline $\begin{array}{c}\text { service } \\
\text { publishing }\end{array}$ & $\begin{array}{l}\text { service } \\
\text { library }\end{array}$ & $\begin{array}{l}\text { service } \\
\text { routing }\end{array}$ & $\begin{array}{c}\text { SOPSA } \\
\text { management }\end{array}$ \\
\hline \multicolumn{2}{|c|}{ service subscription } & \multicolumn{2}{|c|}{ service registration } \\
\hline $\begin{array}{l}\text { safety } \\
\text { service }\end{array}$ & $\begin{array}{l}\text { atabase } \\
\text { ervice }\end{array}$ & $\begin{array}{c}\text { logging } \\
\text { service }\end{array}$ & $\begin{array}{l}\text { context } \\
\text { service }\end{array}$ \\
\hline & \multicolumn{3}{|c|}{ common service } \\
\hline $\begin{array}{c}\text { abstract laye } \\
\text { interface }\end{array}$ & \multicolumn{2}{|c|}{$\begin{array}{l}\text { location } \\
\text { services }\end{array}$} & $\begin{array}{c}\text { LSWSNs } \\
\text { management }\end{array}$ \\
\hline \multicolumn{2}{|c|}{$\begin{array}{c}\text { monitoring and } \\
\text { management service }\end{array}$} & \multicolumn{2}{|c|}{$\begin{array}{c}\text { topology and } \\
\text { configuration services }\end{array}$} \\
\hline & & \multicolumn{2}{|c|}{ SOPA middleware layer } \\
\hline sensor 1 & sensor i & \multicolumn{2}{|c|}{ LSWSNs gateway layer } \\
\hline
\end{tabular}

Figure 1. Reference model of SOPSA
SOPSA solution includes three layers: LSWSNs gateway layer, SOPSA middleware layer, and application service layer. In SOPSA, the upper application can be viewed as a service subscriber or a service provider; the underlying LSWSNs gateway can be regarded as information access. SOPSA achieves the service binding properties by $\mathrm{P} / \mathrm{S}$ mechanism.

The information acquired by LSWSNs gateway enters data networks. Although the sensor data transmission channels vary widely, in large-scale application conditions, the data on a dedicated channel will be stored in a data storage server on public data networks. LSWSNs gateway has the ability to access heterogeneous sensors, can be compatible with multiple interfaces and wireless protocols, has extensible protocol conversion modules, and achieves data collection. LSWSNs gateway can understand the configuration commands of SOPSA middleware layer, docks with the upper platform protocol so as to realize data acquisition, exchange and sensor configuration.

SOPSA middleware is a development platform and infrastructure, which is designed for LSWSN application characteristics. Its main role is to isolate the physical network from the upper application, shields the difference in the communication protocols and data format of different equipment manufacturers so as to provide uniform data processing applications, network monitoring and service delivery interfaces. In order to meet the needs of LSWSNs, SOPSA not only considers the needs of application development, but also considers the characteristics of LSWSNs and the basic capability itself, including the deployment of middleware and the role model. By LSWSNs gateway, SOPSA middleware realizes the configurations for the distributed applications, also can make fine-grained adjustments to the functions of the sensor equipments. By rescheduling tasks and the programmable nodes, SOPSA middleware adjust the relationship between node and gateway. SOPSA achieves this technical requirement in the form of services.

SOPSA middleware includes three layers: LSWSNs management layer, common service layer and SOPSA management layer. LSWSNs management layer includes four modules: topology and configuration services, abstract layer interface, monitoring and management service, and location services. LSWSNs management layer is connected with the LSWSNs gateway, it is responsible for the underlying LSWSNs protocol identification and message processing, and obtains the reliable communication between SOPSA middleware and LSWSNs. Common service layer includes four modules: safety service, database service, logging service, and context service. Common service layer implements the mapping between the perception and the upper layer services, also including the call processing and the coordination between services. SOPSA management layer includes five modules: service publishing, service subscription, service registration, service library, and service routing. Service publishers register, publish services, and process the associated service calls. Service subscribers initiate service queries and calls. Routing service provides a distributed mechanism based on semantic message. According to the service specific information, it transmits the request information to the corresponding role in order for the subsequent processing. 
According to the area of services, service registration is designed to be specialized and distributed. The responsibilities of each service registration include management in both ontology and services, respond to service requests and registration requests. Service registration and service subscription module work together to achieve the service requests which are proposed by the service subscribers.

Application service defines both service interface and application development interface, and supports for application development and service calls from users.

\section{SOPSA MANAGEMENT DESIGN}

SOPSA management layer can intelligently analyze user's requests, and automatically locate specific registration center for appropriate treatments. In this section, we propose a reference framework, shown in Fig .2, to describe the model of SOPSA management layer integrated Pub/Sub mechanism and LSWSNs.

\begin{tabular}{|c|c|c|c|c|c|}
\hline $\begin{array}{l}\text { service } \\
\text { registration }\end{array}$ & \multicolumn{2}{|c|}{$\begin{array}{c}\text { service } \\
\text { composition }\end{array}$} & & \begin{tabular}{|c|} 
service \\
transition
\end{tabular} & $\begin{array}{c}\text { ontology } \\
\text { library }\end{array}$ \\
\hline $\begin{array}{c}\text { service } \\
\text { Qos } \\
\text { selection } \\
\end{array}$ & \multicolumn{2}{|c|}{$\begin{array}{l}\text { service semantic } \\
\text { information } \\
\text { analysis }\end{array}$} & $\leftarrow$ & $\begin{array}{c}\text { service } \\
\text { information } \\
\text { database } \\
\end{array}$ & $\begin{array}{l}\text { service } \\
\text { library }\end{array}$ \\
\hline \multicolumn{6}{|c|}{$\uparrow$} \\
\hline \multicolumn{2}{|c|}{ service routing } & \multicolumn{2}{|c|}{$\begin{array}{c}\text { routing control } \\
\text { management }\end{array}$} & \multicolumn{2}{|l|}{$\begin{array}{c}\text { data } \\
\text { channel }\end{array}$} \\
\hline \multicolumn{4}{|c|}{$\mathfrak{I}$} & \multicolumn{2}{|l|}{$\uparrow$} \\
\hline \multirow{2}{*}{\multicolumn{2}{|c|}{\begin{tabular}{|c|} 
description and \\
submission of \\
service subscription \\
\end{tabular}}} & \multirow{2}{*}{\begin{tabular}{|c|} 
existing \\
services \\
query \\
\end{tabular}} & \multirow{5}{*}{$\leftrightarrow$} & \multicolumn{2}{|c|}{$\begin{array}{c}\text { creation of service } \\
\text { ontology }\end{array}$} \\
\hline & & & & \multirow{2}{*}{\multicolumn{2}{|c|}{\begin{tabular}{|c|} 
service \\
implementation \\
\end{tabular}}} \\
\hline \multirow{2}{*}{\begin{tabular}{|c|} 
service \\
analysis
\end{tabular}} & \multirow{2}{*}{\multicolumn{2}{|c|}{$\begin{array}{c}\text { service } \\
\text { call }\end{array}$}} & & & \\
\hline & & & & \multirow{2}{*}{\multicolumn{2}{|c|}{$\begin{array}{l}\begin{array}{c}\text { service semantic } \\
\text { description }\end{array} \\
\text { service publishing }\end{array}$}} \\
\hline \multicolumn{3}{|c|}{ service subscription } & & & \\
\hline
\end{tabular}

Figure 2. SOPSA management model integrated P/S mechanism and LSWSNs

There are five aspects to the function design about SOPSA management, such as registration, library, routing, subscription and publishing.

\section{A. Registration Function}

Service registration module manages services and responds to both registration requests and service requests. It includes three sub-modules such as service composition, service Qos selection, and service semantic information analysis. The service semantic information analysis submodule resolves service registration requests sent by service providers, and analyses service request information sent by service requestors, it stores the related information into service registry in order to facilitate queries and subsequent calls. The service composition sub-module selects and loads the appropriate discovery and combination algorithm, the candidate services, which are obtained by the algorithm, are combined into a composite service to satisfy the functional requests. The service Qos selection sub-module validates the discovered services. According to QoS and other factors, it removes the services that don't meet service requirements.

\section{B. Publishing Function}

Service registration module completes service publishing. Its main functions include: creating service ontologies, the development and implementation of the services, as well as the publishing and registration of the services. Its function processes are summarized as follows.

- Create ontology. According to the specific industry standards, service providers create practical ontologies, and establish ontology library.

- Upload ontology library. Service providers need registration in the registry center to ensure the service routing can normally navigate to the target registry, and then, upload ontology library files to registration center while the service routing saves a copy of the library files.

- Develop and implement services. Service providers develop and implement the services based ontology, and complete the corresponding test. Ontology-based service development refers to that service information is fully described in accordance with ontology.

- Publish and register services. Service providers publish services, and launch specific service registration requests to the service routing. According to the registration information, the service routing navigates to an appropriate registration center, and manages the services through the registration center. The registration process refers to submitting service description files and information to a registration center.

\section{Subscription Function}

Service subscription module completes service query and service call functions. Its main functions include: description of service requests, submitting service requests, service calls, as well as parsing the results returned by the services. Its function processes are summarized as follows.

- Describe the semantic characteristics of services. Subscriber initiates a service request, subscription module helps users to add and complete the semantic description of the request.

- Query subscription. Service subscription module queries service files in the existing database. If appropriate services are found, the module can directly initiate call, and go to step (4). Otherwise, the module submits the service requests, which are forwarded to the appropriate registration center by the service routing.

- Call service. After the processes of discovery and composition are completed, the registration center returns the sequence of service. The subscription module analyzes the sequence of service, selects the appropriate services, and then initiates a service call. According to the specific service description information, the call module completes these functions, such as service information binding and service return.

\section{TEST AND ANALYSIS}

From two aspects of function and performance, we test the service routing. The test data set is OWL-TC4.0. In the experiment, we choose four different areas of services, such as hotel, event, file and geography. 
Service submission and integration test. In this test, we submit the above four different areas of service files. Service routing handles each of service files respectively. The number of service and processing time are shown in Fig.3.

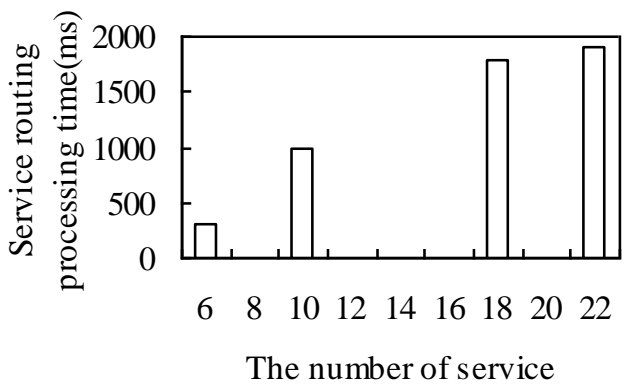

Figure 3. Service submission and integration test

As can be seen from Fig .3, the test results meet the expected situation. Service routing can manage and integrate different service areas. For different areas of registration centers, service routing manages and maps them by service routing table.

Performance test. The test follows the four areas registration center in test 1 . After introducing service routing, we test the response time and return result of indirect connection. At the same time, we build a centralized registration service center, which contains the four different areas of service, to form a direct connection test. The processing times of the service requests are shown in TABLE I.

TABLE I. THE PROCESSING TIMES OF THE SERVICE REQUESTS

\begin{tabular}{|c|c|c|c|}
\hline Test & $\begin{array}{c}\text { Processing Times } \\
\text { of Direct } \\
\text { Connection(ms) }\end{array}$ & $\begin{array}{c}\text { Processing Times } \\
\text { of Indirect } \\
\text { Connection(ms) }\end{array}$ & $\begin{array}{c}\text { Submitted } \\
\text { Concept }\end{array}$ \\
\hline Test 1 & 561 & 892 & city \\
\hline Test 2 & 543 & 853 & text \\
\hline Test 3 & 512 & 866 & longitude \\
\hline
\end{tabular}

As can be seen from table 1 , in the two connection processes, messages have been relocated by service routing, as well as sub-registry center can get results faster.

\section{CONCLUSIONS}

In this paper, we propose SOPSA which is a Pub/Sub architecture for LSWSNs. SOPSA model is defined in a service-oriented environment. We illustrate SOPSA management design through five function aspects. The results show that SOPSA can easily be constructed, and bridge the gap between sensor nodes and LSWSNs applications.

\section{ACKNOWLEDGMENT}

This work was sponsored by open fund project of Jiangsu provincial research and development center of intelligent sensor network engineering technology, china(ZK13-02-03, Software technology, platform and application for sensor network).

\section{REFERENCES}

[1] A. A. A. Alkhatib, and G. S. Baicher, "Wireless Sensor Network Architecture", In International conference on computer networks and communication systems (CNCS 2012), IPCSIT, pp. 11-15.

[2] N. Celandroni, E. Ferro, A. Gotta, G. Oligeri, C. Roseti, M. Luglio, et al. "A Survey of Architectures and Scenarios in Satellite-based Wireless Sensor Networks: System Design Aspects", International Journal of Satellite Communications and Networking, vol. 31(1), 2013, pp. 1-38.

[3] R. S. Alonso, D. I. Tapia, J. Bajo, óscar García, J. F. de Paz, and J. M. Corchado, "Implementing a Hardware-Embedded Reactive Agents Platform based on a Service-oriented Architecture over Heterogeneous Wireless Sensor Networks", Ad Hoc Networks, vol. 11(1), 2013, pp.151-166.

[4] R. Kyusakov, J. Eliasson, and J. Delsing, "Integration of Wireless Sensor and Actuator Nodes with IT Infrastructure using ServiceOriented Architecture", Industrial Informatics, IEEE Transactions, vol. 9(1), 2013, pp. 43-51

[5] L. Mottola, and G. P. Picco, "Programming Wireless Sensor Networks: Fundamental Concepts and State of the Art", ACM Computing Surveys, vol. 43(3), 2011, pp. 19.

[6] A. Lounis, A. Hadjidj, A. Bouabdallah, and Y. Challal, "Secure and Scalable Cloud-based Architecture for e-health Wireless Sensor Networks", In Computer communications and networks (ICCCN 2012), IEEE Press, pp. 1-7.

[7] M. Gholami, N. Cai, and R. W. Brennan, "Evaluating Alternative Approaches to Mobile Object Localization in Wireless Sensor Networks with Passive Architecture", Computers in Industry, vol. 63(9), 2012, pp. 941-947.

[8] N. Ahmad, N. Riaz, and M. Hussain, "Ad hoc Wireless Sensor Network Architecture for Disaster Survivor Detection", International Journal of Advanced Science and Technology, vol. 34, 2011, pp. 9-16.

[9] E. E. Egbogah, and A. O. Fapojuwo, "A Survey of System Architecture Requirements for Health Care-based Wireless Sensor Networks", Sensors, vol. 11(5), 2011, pp. 4875-4898.

[10] Garay J, Guimaraes F, De Oliveira A, et al. Project Wireless Sensor Network Architecture for Tunnel Monitoring[J]. i-ETC: ISEL Academic Journal of Electronics Telecommunications and Computers, 2014, 2(1): ID-18.

[11] B. Koo, K. Han, J. J. J. H. Park, and T. Shon, "Design and Implementation of a Wireless Sensor Network Architecture using Smart Mobile Devices", Telecommunication Systems, vol. 52(4), 2013, pp. 2311-2320.

[12] L. Lamont, M. Toulgoat, M. Deziel, and G. Patterson, "Tiered Wireless Sensor Network Architecture for Military Surveillance Applications", The fifth international conference on sensor technologies and applications (SENSORCOMM 2011), pp.288-294. G. Hackmann, W. Guo, G. Yan, C. Lu, and S. Dyke, "Cyberphysical codesign of distributed structural health monitoring with wireless sensor networks", Parallel and Distributed Systems, vol. 25(1), 2014, pp. 63-72. 